\title{
Evaluation of the Direct Agglutination Test and the rK39 Dipstick Test for the Sero-diagnosis of Visceral Leishmaniasis
}

\author{
Henk DFH Schallig/+ , Marilene Canto-Cavalheiro*, Eduardo S da Silva**
}

\begin{abstract}
KIT (Koninklijk Instituut voor de Tropen/Royal Tropical Institute), Biomedical Research, Meibergdreef 39, 1105 AZ
Amsterdam, The Netherlands *Laboratório de Bioquímica de Tripanosomatídeos, Departamento de Imunologia, Instituto Oswaldo Cruz-Fiocruz, Rio de Janeiro, RJ, Brasil **Laboratório de Leishmanioses, Centro de Pesquisas René Rachou-Fiocruz, Belo Horizonte, MG, Brasil and Centro de Pós-graduação e Pesquisas/Fundação Educacional de Divinópolis, Universidade do Estado de Minas Gerais, Divinópolis, MG, Brasil
\end{abstract}

The direct agglutination test (DAT) based on a freeze-dried antigen and the rK39 dipstick test were evaluated for the sero-diagnosis of visceral leishmaniasis $(V L)$. The sensitivity and specificity of both tests were determined using sera from confirmed VL patients $(n=21)$, healthy controls $(n=19)$ and from patients with other confirmed infectious diseases $(n=42)$. The DAT had a sensitivity and a specificity of 100\%. The rK39 had a sensitivity of $85.7 \%$ and a specificity of $82 \%$. Both tests were also used to screen blood samples of confirmed VL patients $(n=15)$ and serum samples of VL suspects $(n=61)$. The DAT found all blood samples of confirmed VL patients positive and tested $98.4 \%$ of the serum samples of the VL suspects positive. In contrast, rK39 detected in 9/15 blood samples (60\%) antibodies against Leishmania chagasi and found $85.3 \%$ of the serum samples of the suspected patients positive. Although the rK39 dipstick is more rapid and user friendlier than the DAT, the latter has a superior sensitivity and specificity. Furthermore, the reagents used for DAT do not require cold storage, whereas the buffer of the rK39 must be stored at $4^{\circ} \mathrm{C}$. Therefore, the DAT is the most suitable test for the sero-diagnosis of VL under field conditions.

Key words: visceral leishmaniasis - direct agglutination test - rK39 dipstick test - Leishmania chagasi - diagnosis - human - Brazil

Visceral leishmaniasis (VL or kala-azar) is the most severe form of leishmaniasis. Approximately 500,000 new cases of human VL occur annually and the disease is mainly found in Brazil, East Africa and on the Indian subcontinent. American visceral leishmaniasis (AVL), caused by Leishmania chagasi, is a major health problem in many parts of Brazil. Traditionally, the disease was confined to rural and peri-urban areas, but in the last few years the number of human cases of AVL in metropolitan regions is dramatically increasing (Silva et al. 2001). It is important to diagnose AVL as early as possible, because the disease is in most cases fatal if left untreated. The principal clinical symptoms of AVL are an enlarged spleen and a prolonged irregular fever (WHO 1996). Other signs and symptoms are loss of weight, pallor, enlarged liver, enlarged lymph nodes, anaemia, cough and diarrhoea. These signs and symptoms may mimic those of malaria, typhoid, tuberculosis, schistosomiasis and other diseases. The clinical suspicion may be confirmed directly by the detection of parasites in patient material or by culture. However, sample retrieval is inconvenient for the patient and parasite isolation by culture is time-consuming, expensive and difficult.

Because of the above-mentioned limitations of direct diagnostic methods, a number of indirect immunological tests have been developed. These tests should meet several prerequisites in order to be of practical use. They

${ }^{+}$Corresponding author. Fax: +31-20-697.1841. E-mail: H.Schallig@kit.nl

Received 14 December 2001

Acceped 19 June 2002 should be sensitive and specific, cheap (in particular for use in developing countries) and easy to perform under harsh field conditions (Veeken 1999). The direct agglutination test (DAT) remains the first line diagnostic tool in many developing countries as it is a simple test with a high sensitivity, specificity and reproducibility, easyto-perform and not requiring specialised equipment (Zijlstra et al. 1991, Boelaert et al. 1999a,b, Schallig et al. 2001). The introduction of a freeze-dried antigen makes the DAT very suitable for use under remote field conditions as a cold chain for storage of antigen is not required (Meredith et al. 1995, Zijlstra et al. 1997, Schallig et al. 2001). A limitation of the DAT is the relatively long incubation time $(18 \mathrm{~h})$ and the fact that serial dilutions of blood or serum must be made. A dipstick, rK39 dipstick, based on the cloned antigen of a 39 amino acid repeat that is part of a $230 \mathrm{kDa}$ protein encoded by a kinesin-like gene of L. chagasi (Burns et al. 1993), may circumvent these limitations (Sundar et al. 1998).

In the present study, the performance of the DAT based on freeze-dried antigen and commercially available rK39 dipstick in detecting anti-Leishmania antibodies in serum and blood samples from Brazilian individuals is evaluated. Serum samples from patients suffering from a variety of other diseases and healthy controls were also included in the study in order to determine sensitivity and specificity of both tests.

\section{MATERIALS AND METHODS}

Serum and blood samples - Serum samples stored at the serum collection at KIT Biomedical Research (Amsterdam, The Netherlands) were used to determine the sensitivity and specificity of DAT and rK39 under our laboratory conditions. The following panel was used: (1) 
sera from Ethiopian patients ( $\mathrm{n}=10$; a gift from Institute of Pathobiology, University of Addis Ababa, Ethiopia) and Brazilian ( $\mathrm{n}=11$; a gift from the Leishmaniasis Laboratory of the Centro de Pesquisas Rene RachouFiocruz, Belo Horizonte, Minas Gerais, Brazil) with active microscopically proven $\mathrm{VL}(\mathrm{n}=21$; positive control sera); (2) healthy blood donors from Manaus (Amazonas, Brazil) who had no history of VL and no clinical symptoms of leishmaniasis ( $\mathrm{n}=19$; negative control sera); (3) sera from patients with other confirmed diseases: cutaneous leishmaniasis ( $\mathrm{n}=5$ from Belo Horizonte, Minas Gerais); Chagas disease ( $\mathrm{n}=12$ from Recife, Pernambuco, Brazil); African trypanosomiasis ( $\mathrm{n}=5$ from Uganda); leprosy ( $\mathrm{n}=5$ from Indonesia); malaria $(\mathrm{n}=5$ from Kenya); tuberculosis ( $\mathrm{n}=5$ from Kenya); toxoplasmosis ( $\mathrm{n}=5$ from the Netherlands).

Furthermore, the study used clinical samples (serum and blood) of Brazilian individuals. The samples examined are part of the collection at the Leishmaniasis Laboratory of the Centro de Pesquisas Rene Rachou-Fiocruz. All samples were sent to the laboratory under the existing health care system of Metropolitan Region of Belo Horizonte, Minas Gerais, Brazil and stored at $-20^{\circ} \mathrm{C}$. The following two groups of samples were included in the present study: (1) whole blood samples of patients with active VL, microscopically proved $(n=15)$; (2) serum samples of patients clinically suspected of VL, all patients had an enlarged spleen, a prolonged irregular fever and anemia and were living in endemic area $(\mathrm{n}=61)$.

$D A T$ - The DAT was performed essentially as described previously (Meredith et al. 1995, Oskam et al. 1999). In brief, the serum or blood samples were diluted in physiological saline $(0.9 \% \mathrm{NaCl})$ containing $0.8 \% \beta$ mercaptoethanol. Two-fold dilution series of the sera were made, starting at a dilution of 1:100 and going up to a maximum serum dilution of 1:102.400. Freeze-dried DAT antigen ( $L$. donovani promastigotes) produced by KIT Biomedical Research was reconstituted with physiological saline according to the manufacturer's instructions. Fifty $\mu \mathrm{DAT}$ antigen solution (concentration of $5 \times 10^{7}$ parasites per $\mathrm{ml}$ ) was added to each well containing $50 \mu \mathrm{l}$ diluted serum and the results were read after $18 \mathrm{~h}$ of incubation at room temperature. Appropriate control samples with known DAT titres were included as controls. A sample is considered positive if it has a titre $\geq 1: 1600$, the cut-off value of the DAT (ES da Silva, unpublished results).

RK39 dipstick test - Dipsticks were purchased from InBios International (Seattle, USA) and the test was performed according to the manufacturer's instruction. The reading of the test was done after exactly $10 \mathrm{~min}$. A dipstick was considered positive as both the internal control and the test band were stained (irrespective of the intensity of the staining). A dipstick was considered negative as only the internal control was visible. The result of a dipstick was considered not valid if the internal control was not stained.

Reproducibility - Two observers independently read the results of DAT and dipstick assays and their results were compared afterwards. In the case that the interpretation of the dipstick results was different between the two observers or that the reading of the DAT differed more than one serum dilution step the sample was retested in the appropriate test. In the case that the result of the dipstick and the DAT differed from each other, the sample was re-analysed in both tests to confirm the test results.

Determining sensitivity and specificity of the tests The sensitivity (i.e. the probability that the assay will be positive when the infection is present) and the specificity (i.e. the probability that the assay will be negative when the infection is absent) were calculated using the formulas: Sensitivity $=\mathrm{TP} /(\mathrm{TP}+\mathrm{FN}) \times 100 \%$ and Specificity $=$ $\mathrm{TN} /(\mathrm{TN}+\mathrm{FP}) \times 100 \%$. Where $\mathrm{TN}$ represents true negative, TP true positive, FN false negative and FP false positive. The sensitivity of the two tests was assessed with sera from confirmed VL patients $(n=21)$. Sera of healthy controls $(\mathrm{n}=19)$ and sera of patients with confirmed other diseases $(n=42)$ were used to determine the specificity of DAT and rK39 dipstick.

\section{RESULTS}

Reproducibility - Neither the DAT nor rK39 dipstick test had to be repeated due to inconsistencies between the readings of the observers. A $100 \%$ agreement in the reading of the dipstick test and the DAT (one step difference in titre was allowed) was found between the two observers. The analysis performed with rK39 dipstick test and DAT were all valid as the internal control of the dipstick was always positive and the titres of appropriate control samples determined with DAT correspond to the pre-determined titre of the control (one step difference in DAT titre was allowed).

Fourteen samples of which a different result was obtained with DAT and rK39 dipstick were re-tested with both tests and in all cases re-testing resulted in the same test result as initially obtained.

Sensitivity and specificity of DAT and rK39 dipstick test - The results of DAT and rK39 testing of positive and negative controls and of the serum samples of patients with other confirmed infectious diseases are summarised in Table I. Calculation of the sensitivity of the assays revealed that the DAT had a sensitivity of $100 \%$ (no false negative results) and the rK39 had a sensitivity of $85.7 \%$ (three false negative results). None of the negative control samples and samples from patients with other confirmed infectious diseases tested positive with DAT (all titres $<1: 1600 ; 100 \%$ specificity). In contrast, a positive (albeit sometimes faint) reaction was observed with the rk39 dipstick with some of the serum samples of patients with other confirmed infectious diseases (8/42) or healthy endemic controls (3/19). The calculated specificity of the rK39 dipstick on the basis of the results obtained in the present study is $82 \%$.

The DAT found $14 / 15$ blood samples of confirmed VL patients positive $(93.3 \%)$. In contrast, rK39 detected in only 9/15 blood samples (60\%) antibodies against $L$. chagasi.

Evaluation of serum samples of Brazilian VL suspects - The results of DAT and rK39 testing of serum samples of clinical suspects $(n=61)$ are summarised in Table II. The DAT tested $98.4 \%$ of the serum samples of the VL suspects positive, whereas rK39 found $85.3 \%$ of 
the serum samples of the suspected patients positive. Fifty-two samples were positive for both DAT and rK39. Furthermore, DAT was positive for another eight suspects, but these were negative with rK39. Only one sample tested negative with both tests.

\section{TABLE I}

Comparison between direct agglutination test (DAT) and rK39 dipstick using serum samples of confirmed visceral leishmaniasis (VL) patients, blood samples of confirmed VL patients, healthy endemic controls and samples from patients with other confirmed infectious diseases

\begin{tabular}{lcc}
\hline Patient category & Result with DAT & Result with rK39 \\
\hline Serum samples VL patients & $21 / 21$ & $18 / 21$ \\
Blood samples VL patients & $14 / 15$ & $9 / 15$ \\
Healthy controls & $0 / 19$ & $3 / 19$ \\
Cutaneous leishmaniasis & $0 / 5$ & $1 / 5$ \\
Chagas disease & $0 / 12$ & $4 / 12$ \\
African trypanosomiasis & $0 / 5$ & $1 / 5$ \\
Leprosy & $0 / 5$ & $1 / 5$ \\
Malaria & $0 / 5$ & $0 / 5$ \\
Toxoplasmosis & $0 / 5$ & $0 / 5$ \\
Tuberculosis & $0 / 5$ & $1 / 5$ \\
\hline
\end{tabular}

The number represents the number of samples found positive by the test/total number of samples tested.

\section{TABLE II}

Comparison between direct agglutination test (DAT) and rK39 dipstick using serum samples from suspect visceral leishmaniasis patients

\begin{tabular}{lccr}
\hline & rK 39+ & rK 39 - & Total \\
\hline DAT + & 52 & 8 & 60 \\
DAT - & 0 & 1 & 1 \\
\hline Total & 52 & 9 & 61 \\
\hline
\end{tabular}

\section{DISCUSSION}

In the present study, the rK39 test had a lower sensitivity than the DAT based on the freeze-dried antigen. It failed to detect antibodies in three serum samples (one from Ethiopian origin and two from Brazilian origin) of parasitologically confirmed VL patients. This is in line with observations of Zijlstra et al. (2001) who also found a lower sensitivity (67\%) of the rK39 compared to DAT when testing serum samples from African VL patients in Sudan. In addition, Veeken (2001) has observed a low specificity (59-70\%) of the rK39 test. In contrast, Sundar et al. (1998) reported a $100 \%$ sensitivity for the test using peripheral blood samples from Indian patients. This observation was confirmed by Bern et al. (2000) in a study in Nepal. These observations suggest a regional limitation of the use of the rK39 test.

Although not the intended use of the test, according to the manufacturer's instructions, the sensitivity of the rK39 test was also evaluated in the present study with blood samples of confirmed VL patients from the collection at the Leishmaniasis Laboratory of the Centro de Pesquisas Rene Rachou-Fiocruz $(\mathrm{n}=15)$. The sensitivity of rK39 was in this case low, 60\%, whereas DAT found 14 out of the 15 tested samples positive (93.3\% positive).

The specificity of both tests was assessed by using sera from healthy controls and from patients with other confirmed infectious diseases. Several samples of healthy endemic controls tested positive with the dipstick, but not with DAT. This is in line with observations of Zijlstra et al. (2001) who also found healthy Sudanese controls without any history of VL positive with the rK39 dipstick (sensitivity of 96.8\%). More importantly, the rK39 dipstick showed in several cases cross reactions with serum samples of patients with other infectious diseases, among which is Chagas disease (33\% false positives) an infection which in the acute phase should always be included in the differential diagnosis of any unknown fever in the Brazilian situation.

The sensitivity and specificity of the DAT in the present study were determined on a limited number of healthy subjects, patients with visceral leishmaniasis and patients with various other diseases. Therefore $100 \%$ sensitivity and specificity are not claimed and crossreactions can not be fully excluded. It is important to determine the cut-off value of the test using a large as possible number of appropriate local endemic controls (Oskam et al. 1999). Because of the promising results obtained in the present study a large field evaluation of the DAT in different regions of Brazil will be undertaken.

Although the rK39 dipstick has an ideal format for use in the field, as it is a rapid and simple test not requiring extensive training of the operator, its lower sensitivity and specificity limits its use for the sero-diagnosis of VL in Brazil. Furthermore, the test requires cold storage of the running buffer, the test strips can not be stored at high ambient temperatures and the test can not be performed with blood samples. In contrast, the DAT based on freeze-dried antigen does not require cold storage of reagents and can be performed with blood samples. A limitation of the DAT is the relatively long incubation time $(18 \mathrm{~h})$ and the fact that serial dilutions of blood or serum must be made.

Finally, we would like to emphasise that serological tools are an aid in the diagnosis of VL. The outcome of either rK39 dipstick test or DAT should always be judged judiciously in connection with clinical, epidemiological and other diagnostic data.

\section{ACKNOWLEDGEMENTS}

To Professor Asrat Hailu (Institute of Pathobiology, University of Addis Ababa, Ethiopia) for providing us with positive control samples, Dr Birgit van Benthem (KIT Biomedical Research) for critical reading of the manuscript and Nel Kroon (KIT Biomedical Research) and Gerard Schoone (KIT Biomedical Research) for expert technical assistance.

\section{REFERENCES}

Bern C, Jha SN, Joshi AB, Thakur GD, Bista MB 2000. Use of recombinant $\mathrm{k} 39$ dipstick test and the direct agglutination test in a setting endemic for visceral leishmaniasis in Nepal. Am J Trop Med Hyg 63: 153-157. 
Boelaert M, El-Safi SH, Jacquet D, de Muynck A, van der Stuyft P, Le Ray D 1999a. Operational validation of the direct agglutination test (DAT) for diagnosis of visceral leishmaniasis. Am J Trop Med Hyg 60: 129-134.

Boelaert M, El-Safi SH, Mousa H, Githure J, Mbati PA, Gurubacharya VL, Shresta J, Jacquet D, de Muynck A, Le Ray D, van der Stuyft P 1999b. Multi-centre evaluation of repeatability and reproducibility of the direct agglutination test for visceral leishmaniasis. Trop Med Int Health 4: 3137.

Burns JM, Shreffer WG, Benson DR, Ghalib HW, Badaro R, Reed SG 1993. Molecular characterization of a kinesinrelated antigen of Leishmania chagasi that detects specific antibody in African and American visceral leishmaniasis. Proc Natl Acad Sci USA 90: 775-779.

Meredith SEO, Kroon NCM, Sondorp E, Seaman J, Goris MGA, van Ingen CW, Oosting H, Schoone GJ, Terpstra WJ, Oskam L 1995. Leish-KIT, a stable direct agglutination test based on freeze-dried antigen for serodiagnosis of visceral leishmaniasis. J Clin Microbiol 33: 1742-1745.

Oskam L, Nieuwenhuijs JL, Hailu A 1999. Evaluation of the direct agglutination test (DAT) using freeze-dried antigen for the detection of anti-Leishmania antibodies in stored sera from various patient groups in Ethiopia. Trans $R$ Soc Trop Med Hyg 93: 275-277.

Schallig HDFH, Schoone GJ, Kroon CCM, Hailu A, Chappuis F, Veeken H 2001. Development and application of simple diagnostic tools for visceral leishmaniasis. Med Microbiol Immunol 190: 69-72.

Silva ES, Gontijo CMF, Pacheco RS, Fiuza VOP, Brazil RP
2001. Visceral leishmaniasis in the metropolitan region of Belo Horizonte, Sate of Minas Gerais, Brazil. Mem Inst Oswaldo Cruz 96: 285-291.

Sundar S, Reed SG, Singh VP, Kumar PC, Murray HW 1998. Rapid accurate field diagnosis of Indian visceral leishmaniasis. Lancet 351: 563-565.

Veeken H 1999. Manual for the Diagnosis and Treatment of Visceral Leishmaniasis (Kala-Azar) under Field Conditions, Médecins sans Frontières, Amsterdam, The Netherlands.

Veeken H 2001. Control of Infectious Diseases in Developing Countries: Field Studies on Visceral Leishmaniasis and Meningitis, Thesis, University of Amsterdam, The Netherlands.

WHO-World Health Organization 1990. Control of Leishmaniasis. Report of a WHO Expert Committee, Technical Report Series no. 793.

WHO-World Health Organization 1996. Manual on Visceral Leishmaniasis Control, WHO/Leish/96.40.

Zijlstra EE, Ali MS, El Hassan AM, El Toum IA, Satti M, Ghalib HW, Kager PA 1991. Direct agglutination test for diagnosis and sero-epidemiological survey of kala-azar in the Sudan. Trans R Soc Trop Med Hyg 85: 474-476.

Zijlstra EE, Nur Y, Desjeux P, Khalil EAG, El-Hassan AM, Groen J 2001. Diagnosing visceral leishmaniasis with the recombinant K39 strip test: experience from the Sudan. Trop Med Inter Health 6: 108-113.

Zijlstra EE, Osman OF, Hofland HWC, Oskam L, Ghalib HW, El-Hassan AM, Kager PA, Meredith SEO 1997. The direct agglutination test for diagnosis of visceral leishmaniasis under field conditions in Sudan: comparison of aqueous and freezedried antigens. Trans $R$ Soc Trop Med Hyg 91: 671-673. 